\title{
Transplantation of gene-modified haematopoietic stem cells: Application and clinical considerations
}

\author{
M Alessandrini, ${ }^{1} \mathrm{PhD} ; \mathrm{K}-\mathrm{H}$ Krause, ${ }^{1} \mathrm{MD} ; \mathrm{R}$ F Speck, ${ }^{2} \mathrm{MD} ;$ M S Pepper, ${ }^{3} \mathrm{MB} \mathrm{ChB}, \mathrm{PhD}, \mathrm{MD}, \mathrm{PD}$ \\ ${ }^{1}$ Department of Pathology and Immunology, Faculty of Medicine, University of Geneva, Switzerland \\ ${ }^{2}$ Division of Infectious Diseases and Hospital Epidemiology, University Hospital of Zurich, University of Zurich, Switzerland \\ ${ }^{3}$ SAMRC Extramural Unit for Stem Cell Research and Therapy, Institute for Cellular and Molecular Medicine, Department of Immunology, Faculty \\ of Health Sciences, University of Pretoria, South Africa
}

Corresponding author: M S Pepper (michael.pepper@up.ac.za)

\begin{abstract}
Autologous and allogeneic haematopoietic stem cell (HSC) transplantation has been performed in patients with various malignant and non-malignant haematological disorders for more than 50 years. Ex vivo gene modification of HSCs for autologous transplantation opens up new therapeutic avenues for genetic and infectious diseases. Major advances have been made over the last three decades with respect to gene modification of HSCs and transplantation strategies, ultimately culminating in the approval of two such therapies in Europe (Strimvelis for a rare primary immune deficiency, and LentiGlobin for beta-thalassaemia). Newer gene-modifying technologies and treatment regimens have also recently come to the fore, which hold great promise for the development of safer and more effective treatments. We provide an overview of the current state of gene-modified HSC therapies, highlighting success stories, limitations and important considerations for achieving successful translation of these therapies to the clinic.
\end{abstract}

S Afr Med J 2019;109(8 Suppl 1):S65-S70. https://doi.org/10.7196/SAMJ.2019.v109i8b.13910

Genetic modification of haematopoietic stem/progenitor cells (HSCs) for therapeutic applications has been in development for over 30 years, and two such treatments were recently granted market authorisation by the European Medicines Agency. The first, designated GSK2696273 (commercially known as Strimvelis (GlaxoSmithKline (GSK), UK)), is indicated for treatment of a rare primary immune disorder, known as adenosine deaminasedeficient severe combined immunodeficiency (ADA-SCID). ${ }^{[1]}$ Following transplantation of autologous gene-modified HSCs expressing functional ADA, a $100 \%$ survival over a median followup of seven years was shown for 18 study participants. ${ }^{[2]}$ The second treatment, BB305 (Zynteglo, Bluebird Bio, USA), is based on the replacement of functional beta-haemoglobin in HSCs from patients with transfusion-dependent beta-thalassaemia. Data from clinical development show that patients treated with Zynteglo achieved either complete transfusion independence or a near two-thirds reduction in the annual number and volume of blood transfusions. ${ }^{[3]}$ Together with the approval of three other gene modification therapies in 2017 - namely two chimeric antigen receptor (CAR) T cell therapies against $\mathrm{B}$ cell malignancies (axicabtagene ciloleucel (Gilead-Kite Pharma, USA), and tisagenlecleucel (Novartis, Switzerland), as well as Luxturna (Spark Therapeutics, USA)) for an inherited form of vision loss - a new era of modern medicine has been entered into where one can imagine the use of 'single-treatment cures' for life-threatening and previously incurable diseases. The journey to approval of these therapies has by no means been trivial. Notable advances in the technologies and treatment regimens have been made, and credit is due to the scientists, clinicians, institutions and regulators who laid the foundation for future approvals. A description and key aspects relating to successful implementation of HSC gene therapies are highlighted in this review.

\section{Rationale}

Haematopoietic stem cell transplantation (HSCT) is a globally accepted practice for the treatment of malignant and non-malignant disorders of the blood and immune systems. Almost $90 \%$ of HSCTs worldwide are done for the treatment of haematological malignancies, including leukaemia, lymphoma and myeloma. ${ }^{[4,5]}$ For these cases, patients initially receive a chemotherapy regimen to destroy tumour cells, but since the treatment targets all rapidly dividing cells in the body, it also depletes the HSC compartment in the bone marrow. The HSCT is thus aimed at replenishing the bone marrow with stem cells, which engraft and reconstitute the immune system with functional haematopoietic lineages. For non-malignant conditions, primarily rare inherited diseases of the blood and immune systems, the rationale for HSCT is to provide the patient with a haematopoietic lineage that replaces or compensates for the underlying genetic deficiency. Allogeneic HSCT, i.e. transplantation of HSCs harvested from a healthy donor, is essentially the only option for cure of these disorders. This however comes with notable limitations and safety concerns, including the need for a genetically matched donor (which may not be available for up to $70 \%$ of cases), graft rejection, delayed immune reconstitution, graft-versus-host disease and a significant rate of mortality. Autologous transplantation of the patient's own HSCs, which have been gene-modified to correct for the underlying genetic cause of the disease, would thus be the preferred form of treatment for these patients.

\section{Scope of treatment}

Fundamentally, all inherited disorders of the blood and immune systems can be treated with gene-modified HSCs, provided that the underlying genetic cause is known and it is feasible for correction, i.e. gene modification to correct multiple aberrations 
in different genes is presently not feasible. These include primary immune deficiencies, haemoglobinopathies, lysosomal storage and metabolic diseases, and bone marrow failure syndromes - many of which are today treated with allogeneic HSCT. The primary immune deficiencies were the prototype indications treated with gene-modified HSCs. ${ }^{[6]}$ More specifically, these included ADA and X-linked SCID, Wiskott-Aldrich syndrome (WAS) and chronic granulomatosis disease (CGD). Following successful transplantation and engraftment of gene-modified HSCs, together with long-term functional expression of the therapeutic genes, these initial studies provided proof of principle that HSC gene therapy is indeed a rational and feasible treatment approach. In addition to the approvals mentioned previously (Strimvelis (GSK, UK) and Zynteglo (Bluebird Bio, USA)), many such treatments are in pre-clinical and clinical development for various indications (Table 1), and it is estimated that over 200 patients with inherited disorders have been treated to date with gene-modified HSCs.

There is a notable broadening in the scope of treatment, and the transplantation of gene-modified HSCs is also suitable for application in strategies other than the replacement of a defective gene. For example, it is well established that the $\mathrm{C}$ - $\mathrm{C}$ chemokine receptor type 5 (CCR5) co-receptor is a promising target for preventing
HIV infection. In a single case of HIV cure, the so-called Berlin patient received an allogeneic transplantation of HSCs from a CCR5-null donor for reconstitution of his immune system following myeloablative chemotherapy for the treatment of leukaemia. ${ }^{[7]}$ Inspired by these findings, numerous groups, including ours, have developed novel strategies to gene-modify patient HSCs for autologous transplantation and the subsequent generation of an HIVresistant immune system. ${ }^{[8]}$ CCR5 expression can be obviated via gene-silencing approaches, i.e. the integration of short hairpin RNA (shRNA) or microRNA constructs, or the gene itself can be disrupted via gene-editing modalities (see below). In pre-clinical studies, this approach has led to a functional cure of $\mathrm{HIV},{ }^{[9]}$ while clinical studies to assess their safety and efficacy are still underway.

Successful HSC gene therapy ultimately depends on the ability to engraft adequate numbers of gene-modified HSCs that will persist in the long term and express therapeutic genes in all cells of the blood lineages. The treatment procedure is however complex and various aspects require careful consideration, including: (i) the collection of high numbers of HSCs; (ii) safe and efficient cell processing; and (iii) optimal transplantation protocols. Fig. 1 illustrates these concepts, including schematic summaries of relevant gene-modification techniques.

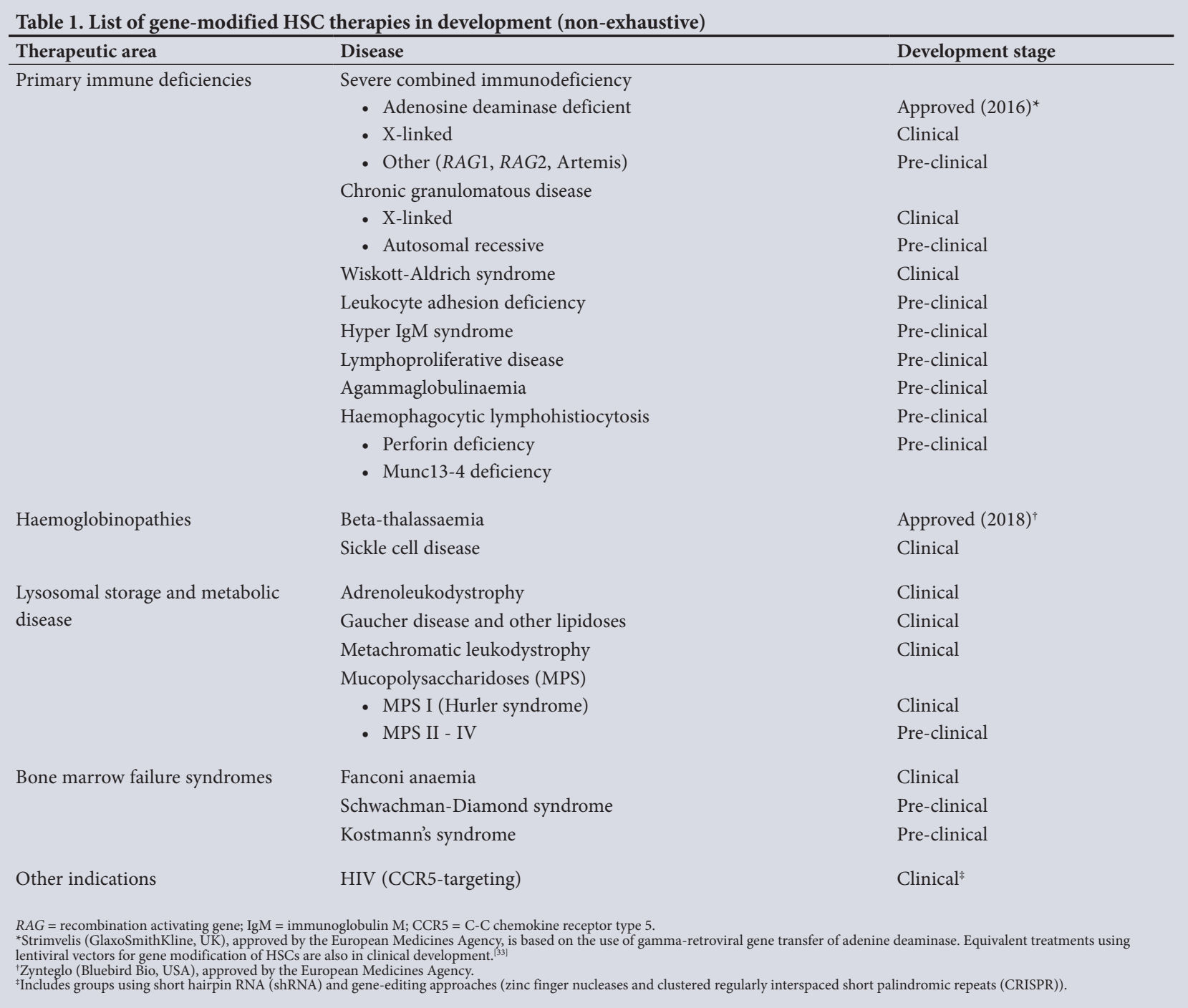




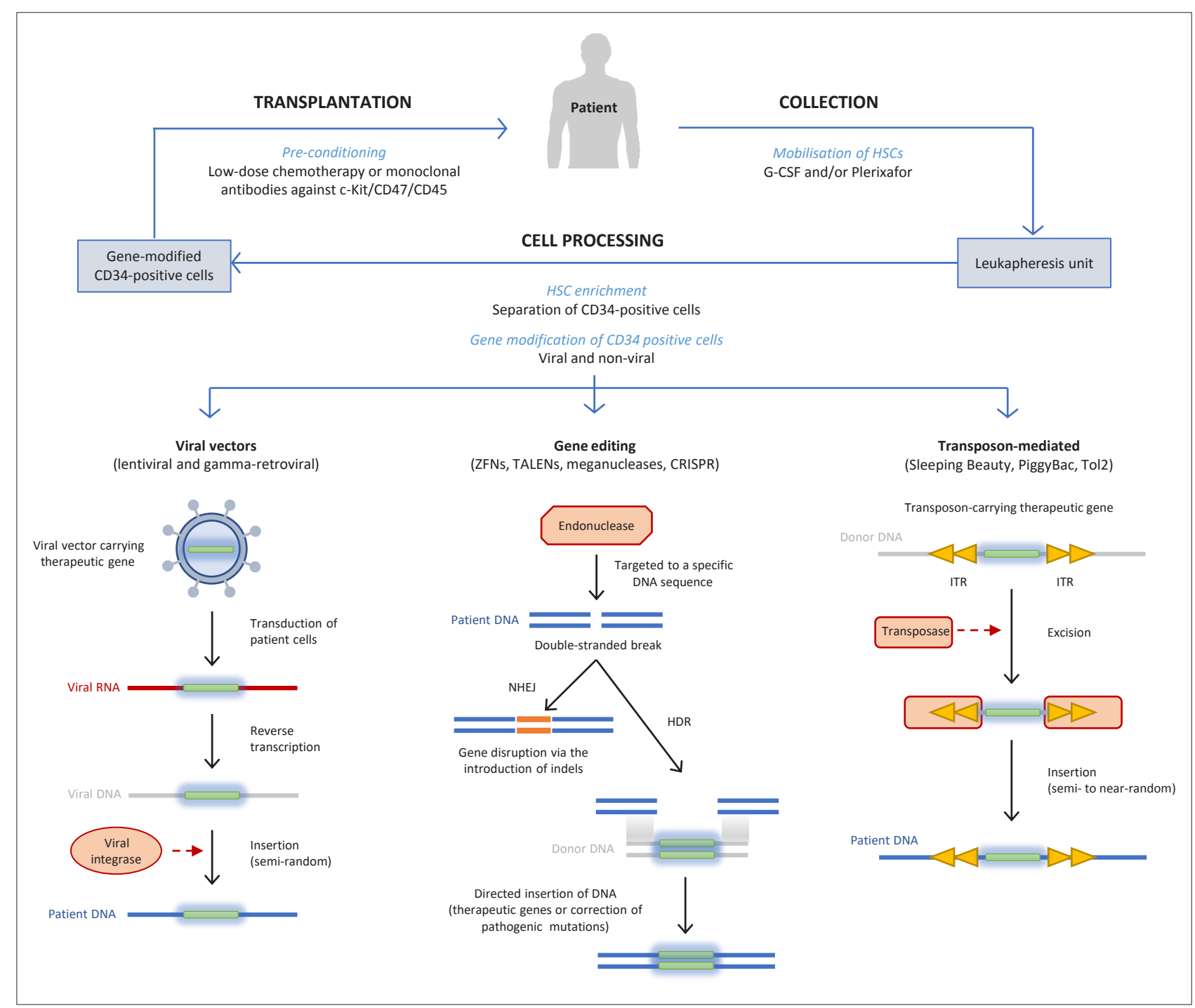

Fig. 1. Haematopoietic stem cell gene therapy. Considerations for the collection, cell processing and transplantation are provided. A schematic overview of the different approaches used to gene-modify patient cells is also provided, namely the use of viral vectors and non-viral approaches (gene editing and transposonmediated). (CRISPR = clustered regularly interspaced short palindromic repeats; G-CSF = granulocyte-colony-stimulating factor; HDR $=$ homology-directed repair; HSC = haematopoietic stem and progenitor cells; ITR = inverted terminal repeat; NHEJ = non-homologous end joining; TALENs = transcription activator-like effector nucleases; ZFN = zinc finger nucleases.)

\section{Collection of HSCs}

HSCs are multipotent stem cells, generally defined by their expression of the CD34 antigen, and mainly reside in the bone marrow, where conditions are optimal for haematopoiesis. Since HSCs constitute less than $0.1 \%$ of cells in peripheral circulation, they have traditionally been harvested directly from the bone marrow via the iliac crest. As an alternative, HSCs can be harvested from the peripheral blood following treatment with haematopoietic growth factors, such as granulocyte-colony-stimulating factor (G-CSF; also known as neupogen or filgrastim), which stimulate HSC cycling and thereby significantly increase numbers in the peripheral blood. In Europe and the US, $~ 90 \%$ of HSCTs are performed using peripheral blood mobilised HSCs. ${ }^{[4,5]}$ In a typical HSC collection from peripheral blood, donors will receive G-CSF twice daily over 4 - 5 days, followed by harvesting via leukapheresis. A leukapheresis unit contains all the white blood cells, which have been separated from plasma and red blood cells based on density centrifugation. In addition to the CD $34^{+}$HSC population, the white blood cell compartment contains lymphocytes, granulocytes and monocytes.
It is important that adequate numbers of $\mathrm{CD} 34^{+}$cells are harvested from the patient to compensate for inevitable losses in the downstream processing and preparation of the final drug product. A patient collection should thus yield over 6 million CD $34^{+}$cells per $\mathrm{kg}$ to ensure that a minimum number of 3 - 5 million genemodified cells per $\mathrm{kg}$ are finally re-introduced to the patient. This is often achieved in patients mobilised with G-CSF, but not in all cases, such as in those with sickle cell disease, ${ }^{[10]}$ where HSCs mobilise rather poorly with G-CSF, which results in an insufficient number of $\mathrm{CD}_{3} 4^{+}$cells being collected. More recently, plerixafor (an inhibitor of C-X-C chemokine receptor type 4 (CXCR4), a receptor responsible for the homing of HSCs to the bone marrow) has been used to either complement G-CSF mobilisation or on its own, which has resulted in substantial increases in $\mathrm{CD}^{2} 4^{+}$stem cell harvests. Plerixafor has a favourable safety profile and requires either a single dose (or at most one additional dose) to achieve CD $34^{+}$cell mobilisation in adequate numbers. ${ }^{[11]}$ Even so, in cases such as Fanconi anaemia it is still difficult to obtain adequate numbers of $\mathrm{CD} 34^{+}$cells. ${ }^{[12]}$ 


\section{Cell processing}

The leukapheresis unit, containing all white blood cells, carries up to 60 billion cells in volumes of up to $400 \mathrm{~mL}$. The initial processing of patient cells includes separation of the $\mathrm{CD} 34^{+}$population using anti-CD34 antibody bound to paramagnetic particles/beads. Once added to the leukapheresis unit, the beads bind to cells expressing extracellular CD34, which can then be positively selected for using a magnet and a series of washing procedures to first remove all the unwanted cells (monocytes, granulocytes and other lymphocytes) and then to dissociate the magnetic beads from the enriched $\mathrm{CD} 34^{+}$population. For clinical grade and scale cell separation, specific equipment such as the CliniMACS (Miltenyi Biotec, Germany) is required, which is reported to yield recovery rates of $\sim 70 \%$ and purities of over $95 \% .{ }^{[13]}$ The CliniMACS Prodigy, a next-generation device, has additional functionalities that facilitate gene modification of patient cells, making it the preferred instrument for gene therapy applications. These devices are certified for cell therapy applications and globally accepted by regulatory authorities. ${ }^{[14,15]}$ However, the system is costly and has low throughput, which will have its challenges if we are to foresee roll-out of ex vivo gene-modified cell therapies for diseases such as HIV and sickle cell disease where the patient burden is in the millions and is often in lower-to-middle income countries. There is thus an opportunity to address these limitations with more efficient process developments, lower cost and higher throughput equipment solutions.

Broadly speaking, two strategies for gene modification of HSCs are applied, namely viral and non-viral (Table 2). Viral vectors (in particular lentiviral and gamma-retroviral vectors) are well-established technologies, and both the approved HSC gene therapies (Strimvelis and LentiGlobin) make use of these approaches for gene modification of patient cells. The recently approved chimeric antigen receptor (CAR) $\mathrm{T}$ cell therapies also make use of viral vectors. ${ }^{[16]}$ Although far less well established, nonviral approaches comprising gene editing and transposon-based technologies are believed to hold great promise for future clinical applications.

\section{Viral vector gene modification}

Viral vectors form the cornerstone of gene-modified cell therapies. They are derived from natural viruses, but have been reconstructed to infect and optimally deliver genetic material to target cells. To make them clinically viable, several elements of the viral genome have been adapted to render them self-inactivating, which prevents viral propagation. Many varieties of viral vectors exist, some of which allow for integration of therapeutic genes (e.g. retroviral and lentiviral), while others deliver genetic material for a transient effect (e.g. adeno- and adeno-associated viral vectors). The latter group of vectors is also employed by gene-editing and transposon modalities for transient delivery of their respective components (see non-viral gene modification section below).

For integration of therapeutic genes, gamma-retroviral and lentiviral vectors are mainly used, with the latter being the vector of choice for gene modification of cells of the haematopoietic system. Following transduction (infection) of patient cells, the viral RNA is converted to DNA by reverse transcriptase and then integrated into the cellular genome in a semi-random fashion using the integrase enzyme provided by the viral vector. The primary concern for using viral vectors is one of safety, in particular insertional mutagenesis. Initial clinical studies showed that HSC gene therapy using gamma-retroviral vectors was curative for the majority of primary immune deficiency patients (with ADA-SCID, X-SCID, WAS and CGD), but they were later marred by the occurrence of treatment-related haematological malignancies. After 2 years of follow-up, $25 \%$ of the patients presented with myelodysplasia or leukaemia due to insertional mutagenesis caused by the gammaretroviral vectors. ${ }^{[6]}$ Essentially, gamma-retroviral vectors typically insert near proto-oncogenes, which resulted in their activation and malignant transformation of the gene-modified cells. Interestingly, with over 45 patients treated to date, no ADA-SCID patients receiving gene therapies with gamma-retroviral vectors have thus far developed malignancies. The reasons for this remain unclear.

Following these studies, numerous advances in viral vectormediated gene transfer have been made, including the implementation and acceptance of lentiviral vectors as the vector of choice. ${ }^{[17]}$

Table 2. Advantages and disadvantages of gene transfer systems used for gene modification of HSCs

\begin{tabular}{ll}
\hline System & Advantages \\
\hline Viral vectors $^{*}$ & Established technology and the basis of all currently \\
& approved ex vivo gene-modified cell therapies \\
& Favourable safety profile (generally accepted for \\
& lentiviral vectors) \\
& High efficiency gene modification of HSCs (following \\
& protocol optimisation)
\end{tabular}

Gene editing ${ }^{\ddagger} \quad$ Targeted (non-random) editing of cells Versatile - allows for gene insertion, correction of pathogenic mutations and gene disruption Disadvantages

Risk of insertional mutagenesis (when using gammaretroviral vectors)

Laborious and costly to manufacture protocol optimisation)

\begin{tabular}{|c|c|c|}
\hline हैก & $\begin{array}{l}\text { Versatile - allows for gene insertion, correction of } \\
\text { pathogenic mutations and gene disruption }\end{array}$ & Risk of off-target effects and cellular toxicity \\
\hline & & $\begin{array}{l}\text { Low efficiency gene modification of HSCs } \\
\text { Laborious and costly to manufacture }{ }^{\S}\end{array}$ \\
\hline Transposons & $\begin{array}{l}\text { Low immunogenicity } \\
\text { Affordable to manufacture }\end{array}$ & $\begin{array}{l}\text { New technology with limited use in clinical studies } \\
\text { Low efficiency gene modification of HSCs } \\
\text { Risk of off-target effects and cellular toxicity }\end{array}$ \\
\hline
\end{tabular}


Lentiviral vectors are associated with low immunogenicity and have been shown to integrate more randomly into the genome without risk of insertion near proto-oncogenes. In comparison with gammaretroviral vectors, it has been shown that lentiviral transduction of HSCs results in integration at over 1500 insertion sites relative to less than 100 for gamma-retroviral vectors. ${ }^{[18,19]}$ The most important conclusion to be made from these studies is that lentiviral vectors are far less prone to interfering with genes which result in HSC transformation. The situation is thus entirely different for lentiviral vectors, with no cases of insertional mutagenesis being reported to date. Notably, HIV belongs to the family of lentiviruses, and even with all the cases of HIV infection recorded to date, there have been no reports of haematological malignancies due to insertional mutagenesis.

Additional benefits of lentiviral vectors are that they are able to infect a broader range of target cells, including non-dividing cells. The latter is particularly relevant in the context of HSCs, which typically transduce poorly when compared with other cells of the haematopoietic system, such as T cells. Even with lentiviral vectors, transduction rates can be as low as $10 \%$ in $\mathrm{CD} 34^{+}$cells compared with $>90 \%$ in $\mathrm{T}$ cells when using equivalent ratios of vector to target cells (referred to as multiplicity of infection (MOI)). Optimisation of lentiviral transduction protocols over the years has resulted in significant improvements in transduction rates, including the use of high MOIs (>100), transduction over 2 consecutive days, optimised growth media and cytokine usage, ${ }^{[18-20]}$ and transduction enhancers. ${ }^{[21,22]}$

\section{Non-viral gene modification}

Gene editing also allows for the insertion of a therapeutic gene, but with the added advantage of being able to do so in a targeted fashion. The technology is based on a joint delivery of guiding elements (DNA binding) and an endonuclease enzyme, which allow one to target and cut genomic DNA in a highly specific manner. In addition to gene insertion, the ability to direct endonuclease activity means that gene editing further allows for the correction of genes at the site of a pathogenic mutation or the disruption of therapeutically relevant genes (such as CCR5 for HIV). Following a targeted double- stranded break of DNA, gene-editing techniques rely (and capitalise) on the natural mechanisms of DNA repair in the cell. Homology-directed repair (HDR) enables insertion of genetic material, which can be entire therapeutic genes or shorter fragments for correction of pathogenic mutations. Gene disruption relies on non-homologous end joining (NHEJ) to simultaneously integrate small insertions or deletions into a target gene, thereby resulting in a shift in the reading frame and subsequent loss of function. Therefore, relative to viral vector gene modification, gene editing is a more versatile technology by virtue of it being a site-directed modality.

Four broadly categorised mechanisms of gene editing exist, namely zinc finger nucleases (ZFNs), transcription activator-like effector nucleases (TALENs), meganucleases and clustered regularly interspaced short palindromic repeats (CRISPR)-associated Cas. Each varies mechanistically in the way it targets and edits genomic DNA, construction and manufacturing complexity, as well as in editing efficiencies. ${ }^{[23]}$ In the context of HSC gene modification, gene-editing techniques are generally poorly efficient relative to viral vectors. These new technologies are still undergoing 'growing pains' and key safety issues are still to be resolved before clinical application can unequivocally be considered viable. The most notable challenge is the risk of off-target effects, which could result in functional impairment or decreased fitness of edited cells, or more relevantly introduce pro-oncogenic risks. ${ }^{[23,24]}$
Transposon-based systems make up the second class of non-viral gene-modification strategies. Transposons are naturally occurring and mobile DNA elements that have the ability to change their positions in the genome. Within their structure, they have a recognition domain for transposase - the enzyme performing the 'cut and paste' function - and are flanked by inverted terminal repeat sequences. Once the DNA element is excised, it is re-inserted in a semi-random fashion across the genome. ${ }^{[25]}$ For therapeutic application, a transposon carrying the therapeutic gene is provided by a synthetic DNA donor strand. Following excision via transposase, the transposon is inserted in a semi-random manner in the genome of patient cells. Three transposon-based systems have been developed for therapeutic purposes, namely Sleeping Beauty, PiggyBac and Tol2. Each differ in their origins and have specific advantages and disadvantages. Sleeping Beauty seems to integrate in a closer-torandom manner across the genome, whereas the other two tend to integrate in a profile similar to that of gamma-retroviral vectors. ${ }^{[26,27]}$ PiggyBac and Tol2 have the ability to carry larger genetic cargos, while PiggyBac is the most efficient with the highest transposition activity.

At present, transposon-mediated gene modification is still relatively inefficient, and as is the case for viral vectors and gene editing modalities, gene transfer to HSCs remains the biggest challenge. Significant advances have been made recently, ${ }^{[25,27]}$ and considering the advantages and simplicity of the technology, it is foreseen that this will remain a promising alternative and part of the future landscape of gene-modified cell therapies. Notably, a transposon-based system is being successfully applied for creating CAR T cells, with development being in clinical trial stages. ${ }^{[25,28]}$

\section{Transplantation}

'Conditioning' of patients prior to HSCT is essential for successful engraftment of HSCs. The principle is based on the premise of creating a niche within the bone marrow (by depleting proliferating HSCs) where the transplanted HSCs are able to home and engraft. Various conditioning regimens exist and the decision on which to use depends on the disease being treated, the stage of the disease and the patient's medical status. Conditioning regimens can be in the form of total body irradiation and/or cytotoxic/chemotherapeutic agents, and can be administered at high doses to achieve maximal ablation (myeloablation) of the bone marrow or at low doses for reduced-intensity conditioning (RIC) purposes. For allogeneic HSCT, the conditioning regimens may also include immunosuppressive treatments to prevent graft rejection or the risk of graft-versus-host disease. With respect to transplantation of autologous gene-modified HSCs, RIC is almost always indicated, except in certain cases (such as SCID), where the gene-modified cells have a significant survival advantage. ${ }^{[6]}$

The morbidity associated with conditioning regimens is not trivial: in the short term, patients suffer complications such as mucositis, neutropenia and a high risk of infection, while in the long-term risks of sterility, organ damage and secondary tumours can be anticipated. Given these safety concerns, several groups have started to develop alternative pre-conditioning regimens, which aim to be 'chemo-free' and with significantly improved safety profiles without a compromise with regard to HSC engraftment. Moreover, such alternatives would provide welcome relief to groups developing genemodified HSC treatments for less severe diseases such as HIV, where cytotoxic conditioning regimens pose a significant challenge for the feasibility of their treatments. Novel approaches in this regard include the use of immunotoxins and monoclonal antibodies directed to markers expressed on HSCs, such as c-Kit, ${ }^{[29]} \mathrm{c}-\mathrm{Kit}$ and $\mathrm{CD} 47,{ }^{[30]}$ and 
CD45. ${ }^{[31]}$ Impressive HSC depletion in pre-clinical models of up to 99\% has been reported. ${ }^{[30]}$ More recently, CAR T cells against the c-Kit antigen on HSCs have also been reported as a promising approach. ${ }^{[32]}$ Although these are encouraging preclinical developments, thorough clinical testing is still required - we have only been able to identify one registered clinical trial in which a monoclonal antibody against c-Kit is being used as pre-conditioning in SCID patients receiving allogeneic HSCT (NCT02963064).

\section{Conclusion}

The recent approval of ex vivo gene-modified HSC therapies is the culmination of decades of work with significant improvements in vector technologies and HSCT treatment regimens. These treatments are today accepted by the global medical community, and provide a new paradigm of curative treatments. However, several improvements can still be made to achieve optimal safety and efficacy parameters. Gene-editing and transposon systems offer a new dimension for genetic modification of patient cells, and hold great promise for future application for a variety of diseases. Another hopeful prospect is the potential for chemotherapy-free conditioning, which would substantially improve treatment quality and lower the barrier to entry for novel therapy approvals. Finally, a notable consideration for routine application of these treatments is around the topic of cost. The approved Strimvelis and Zynteglo therapies cost in excess of USD500 000 ( ZAR7 million), which is the result of the cost of viral vector manufacturing, cell processing and the application of these treatments as personalised therapies. With the future scalingup of these treatments, costs are anticipated to decrease over time, but are likely to remain unaffordable to the majority of patients in low- to middle-income countries. Innovative strategies are required to enable global access to these treatments, and to ensure that patients in resource-poor communities are not excluded from these exciting developments.

\section{Acknowledgments. None.}

Author contributions. MA: Manuscript writing; KHK and RS: Manuscript editing; MSP: Conception, manuscript editing, project management, financial support.

Funding. Work in MSP's group is funded by the South African Medical Research Council (University Flagship and Extramural Unit awards). RFS is supported by the Clinical Research Priority Program (CRPP), Human Hemato-Lymphatic Diseases, of the University of Zurich.

Conflicts of interest. None.

1. Aiuti A, Roncarolo MG, Naldini L. Gene therapy for ADA-SCID, the first marketing approval of an ex vivo gene therapy in Europe: Paving the road for the next generation of advanced therapy medicinal products. EMBO Mol Med 2017;9(6):737-740. https://doi.org/10.15252/emmm.20170757

2. Cicalese MP, Ferrua F, Castagnaro L, et al. Update on the safety and efficacy of retroviral gene therapy for immunodeficiency due to adenosine deaminase deficiency. Blood 2016;128(1):45-54. https://doi org/10.1182/blood-2017-05-782862
3. Thompson AA, Walters MC, Kwiatkowski J, et al. Gene therapy in patients with transfusion-dependent beta-thalassemia. N Engl J Med 2018;378(16):1479-1493. https://doi.org/10.1056/nejmoal705342 . Passweg JR, Baldomero $\mathrm{H}$, Bader $\mathrm{P}$, et al. Is the use of unrelated donor transplantation leveling off in Europe? The 2016 European Society for Blood and Marrow Transplant activity survey report. Bone
Eussweg IR Europe? The 2016 European Society for Blood and Marrow Transplant activity survey
Marrow Transplant 2018;53(9):1139-1148. https://doi.org/10.1038/s41409-018-0153-1

5. Currew Transplant 2018;53(9):1139-1148. https:///doi.org/10.1038/s41409-018-0153-1
5ses and Outcomes of Haematopoietic Cell Transplantation (HCT): CIBMTR Summary Slides. 5. Current Uses and Outcomes of Haematopoietic Cell
http://www.cibmtr.org (accessed 10 December 2018).

http://www.cibmtr.org (accessed 10 December 2018).
6. Booth C, Gaspar HB, Thrasher AJ. Treating immunodeficiency through HSC gene therapy. Trends Mol 6. Booth C, Gaspar HB, Thrasher AJ. Treating immunodeficiency through
Med 2016;22(4):317-327. https://doi.org/10.1016/j.molmed.2016.02.002

7. Hutter G, Nowak D, Mossner M, et al. Long-term control of HIV by CCR5 Delta32/Delta32 stem-cell transplantation. N Engl J Med 2009;360(7):692-698. https://doi.org/10.1056/NEJMoa0802905.

8. Haworth KG, Peterson CW, Kiem HP. CCR5-edited gene therapies for HIV cure: Closing the door to viral entry. Cytotherapy 2017;19(11):1325-1338. https://doi.org/10.1016/j.jcyt.2017.05.013

9. Myburgh R, Ivic S, Pepper MS, et al. Lentivector knockdown of CCR5 in haematopoietic stem and progenitor cells confers functional and persistent HIV-1 resistance in humanized mice. J Virol 2015;89(13):6761-6772. https://doi.org/10.1128/jvi.00277-15

10. Lagresle-Peyrou C, Lefrère F, Magrin E, et al. Plerixafor enables safe, rapid, efficient mobilization of haematopoietic stem cells in sickle cell disease patients after exchange transfusion. Haematologica 2018;103(5):778-786. https://doi.org/10.3324/haematol.2017.184788

11. Pantin J, Purev E, Tian X, et al. Effect of high-dose plerixafor on CD34(+) cell mobilization in healthy stem cell donors: Results of a randomized crossover trial. Haematologica 2017;102(3):600-609. https:// doi.org/10.3324/haematol.2016.147132

12. Adair JE, Sevilla J, Heredia CD, Becker PS, Kiem HP, Bueren J. Lessons learned from two decades of clinical trial experience in gene therapy for Fanconi anemia. Curr Gene Ther 2017;16(5):338-348. https://
cing doi.org/10.2174/1566523217666170119113029

13. Schumm M, Lang P, Taylor G, et al. Isolation of highly purified autologous and allogeneic peripheral CD34+ cells using the CliniMACS device. J Hematother 1999;8(2):209-218. https://doi. peripheral CD34+ cells using

14. Hümmer C, Poppe C, Bunos M, et al. Automation of cellular therapy product manufacturing: Results of a split validation comparing $\mathrm{CD} 34$ selection of peripheral blood stem cell apheresis product with a semimanual vs. an automatic procedure. J Transl Med 2016;14:76. https://doi.org/10.1186/s12967-016-0826-8

15. Stroncek DF, Tran M, Frodigh SE, et al. Preliminary evaluation of a highly automated instrument for the selection of $\mathrm{CD} 34+$ cells from mobilized peripheral blood stem cell concentrates. Transfusion 2016;56(2):511-517. https://doi.org/10.1111/trf.13394

16. Tran E, Longo DL, Urba WJ. A milestone for CAR T cells. N Engl J Med. 2017;377(26):2593-2596. https:// doi.org/10.1056/nejme1714680

17. Cesana D, Volpin M, Serina Secanechia YN, Montini E. Safety and efficacy of retroviral and lentiviral vectors for gene therapy. In: Brunetti-Pierri N, ed. Safety and Efficacy of Gene-Based Therapeutics for Inherited Disorders. Cham: Springer, 2017:9-35.

18. Aiuti A, Biasco L, Scaramuzza S, et al. Lentiviral haematopoietic stem cell gene therapy in patients with Wiskott-Aldrich syndrome. Science 2013;341(6148):1233151. https://doi.org/10.1126/science.1233151

19. Cartier N, Hacein-Bey-Abina S, Bartholomae CC, et al. Lentiviral haematopoietic cell gene therapy for X-linked adrenoleukodystrophy. Methods Enzymol 2012;507:187-198. https://doi.org/10.1016/b978-012-386509-0.00010-7

20. Biffi A, Montini E, Lorioli L, et al. Lentiviral haematopoietic stem cell gene therapy benefits metachromatic leukodystrophy. Science 2013;341(6148):1233158.

21. Piovan C, Marin V, Scavullo C, et al. Vectofusin-1 promotes RD114-TR-pseudotyped lentiviral vector transduction of human HSPCs and T lymphocytes. Mol Ther Methods Clin Dev 2017;5:22-30. https:// doi.org/10.1016/j.omtm.2017.02.003

22. Heffner GC, Bonner M, Christiansen L, et al. Prostaglandin E2 increases lentiviral vector transduction efficiency of adult human haematopoietic stem and progenitor cells. Mol Ther 2018;26(1):320-328. https://doi.org/10.1016/j.ymthe.2017.09.025

23. Cox DB, Platt RJ, Zhang F. Therapeutic genome editing: Prospects and challenges. Nature Med 2015;21(2):121-131. https://doi.org/10.1038/nm.3793

24. Maeder ML, Gersbach CA. Genome-editing technologies for gene and cell therapy. Mol Ther 2016;24(3):430-446. https://doi.org/10.1038/mt.2016.10

25. Kebriaei P, Izsvak Z, Narayanavari SA, Singh H, Ivics Z. Gene therapy with the Sleeping Beauty transposon system. Trends Genet 2017;33(11):852-870. https://doi.org/10.1016/j.tig.2017.08.008

26. Huang X, Guo H, Tammana S, et al. Gene transfer efficiency and genome-wide integration profiling of Sleeping Beauty, Tol2, and piggyBac transposons in human primary T cells. Mol Ther 2010;18(10):18031813. https://doi.org/ $10.1038 / \mathrm{mt} 2010.141$

27. Holstein M, Mesa-Nunez C, Miskey C, et al. Efficient non-viral gene delivery into human haematopoietic stem cells by minicircle Sleeping Beauty transposon vectors. Mol Ther 2018;26(4):1137-1153. https://doi. org/10.1016/j.ymthe.2018.01.012

28. Kebriaei P, Singh H, Huls MH, et al. Phase I trials using Sleeping Beauty to generate CD19-specific CAR T cells. J Clin Invest 2016;126(9):3363-3376. https://doi.org/10.1172/cici86721

29. Czechowicz A, Kraft D, Weissman IL, Bhattacharya D. Efficient transplantation via antibody-based . Czechowicz A, Kraft D, Weissman I, Bhattacharya D. Efficient transplantation via antibody-based
clearance of haematopoietic stem cell niches. Science 2007;318(5854):1296-1299. https://doi.org/10.1126/ science. 1149726

30. Chhabra A, Ring AM, Weiskopf $\mathrm{K}$, et al. Haematopoietic stem cell transplantation in immunocompetent hosts without radiation or chemotherapy. Sci Transl Med 2016;8(351):351ra105. https://doi.org/10.1126/ hosts without radiation

31. Palchaudhuri R, Saez B, Hoggatt J, et al. Non-genotoxic conditioning for haematopoietic stem cell transplantation using a haematopoietic-cell-specific internalizing immunotoxin. Nature Biotechnol 2016;34(7):738-745. https://doi.org/10.1038/nbt.358

32. Arai Y, Choi U, Corsino CI, et al. Myeloid conditioning with c-Kit-targeted CAR-T cells enables donor stem cell engraftment. Mol Ther 2018;26(5):1181-1197. https://doi.org/10.1016/..ymthe.2018.03.003

33. Gaspar HB, Buckland K, Carbonaro DA, et al. C-8. Immunological and metabolic correction after lentiviral vector gene therapy for ADA deficiency. Mol Ther 2015;23(Suppl 1):S102-S103. 